DOI: $10.17516 / 1997-1397-2020-13-3-275-284$

УДК 519.21

\title{
On Limit Theorem for the Number of Vertices of the Convex Hulls in a Unit Disk
}

\author{
Isakjan M. Khamdamov* \\ Tashkent University of Information Technologies \\ Tashkent, Uzbekistan
}

Received 12.02.2020, received in revised form 06.03.2020, accepted 03.04.2020

\begin{abstract}
This paper is devoted to further investigation of the property of a number of vertices of convex hulls generated by independent observations of a two-dimensional random vector with regular distributions near the boundary of support when it is a unit disk. Following P. Groeneboom [4], the Binomial point process is approximated by the Poisson point process near the boundary of support and vertex processes of convex hulls are constructed. The properties of strong mixing and martingality of vertex processes are investigated. Using these properties, asymptotic expressions are obtained for the expectations and variance of the vertex processes that correspond to the results previously obtained by H. Carnal [2]. Further, using the properties of strong mixing of vertex processes, the central limit theorem for a number of vertices of a convex hull is proved.
\end{abstract}

Keywords: convex hull, Poisson point process, Markovian jump process, martingales, Central limit theorem.

Citation: I.M.Khamdamov, On Limit Theorem for the Number of Vertices of the Convex Hulls in a Unit Disk, J. Sib. Fed. Univ. Math. Phys., 2020, 13(3), 275-284.

DOI: $10.17516 / 1997-1397-2020-13-3-275-284$

\section{Introduction}

The functionals of convex hulls are complex objects in analytical aspect. Therefore, studying the properties of even the simplest functionals of convex hulls such as the number of vertices or the area, has for a long time remained a difficult task. This explains the fact that such wellknown researchers as in $[2,3,15]$ and others, limited their interests to studying the average value of the number of vertices, the area, and the perimeter of a random polygon. For many years, due to the lack of valid research methods, the attempts to develop this area have not been successful.

In paper [4] has made a significant progress in this field. He managed for the first time to obtain the limit distribution for the number of vertices of a convex hull in the case when the support of initial uniform distribution is either a convex polygon or an ellipse. His research method is based on the original idea of using the Poisson approximations of a binomial point process near the boundary of the support of initial distribution. Then he applied powerful methods such as martingales, mixing of stationary processes and others. Based on this method, in [1] have established the limiting distribution for the area of the convex hull when the support of initial distribution is a convex polygon. In [6] proved the limit theorems for the area outside a convex hull when the support is a unit disk. These results in a more general form, for the

*khamdamov.isakjan@gmail.com https://orcid.org/0000-0002-7464-8358

(c) Siberian Federal University. All rights reserved 
joint distribution of the vertex number, area, and perimeter, were obtained by [12] using the idea of [4] on Poisson approximations of a binomial point process near a polygon boundary. In [7] has developed this problem for the case when the convex hull is generated by distributions with exponential tails, including, in particular, the normal distribution.

The approach used in this paper is a modification of the methods proposed by $[4,5,12]$ and adapted to a wider class of initial distributions.

\section{Statement of the problem and results formulation}

Let the support of initial distribution $A$ be a unit disk with a center at a point $(0,1)$.

Suppose that random points $\left(r_{i}, \alpha_{i}\right)$ are given in the polar coordinate system (with pole $(0,1)$ ) in a disk $A$, where $r_{i}$ and $\alpha_{i}$ are independent and $\alpha_{i}$ is uniformly distributed in $[-\pi, \pi]$ and

$$
P\left(r_{i}>1-x\right)=x^{\beta} L\left(\frac{1}{x}\right), 0<x<1, \beta \geqslant 1,
$$

where $L(x)$ is the slowly varying function in the Karamata sense given by

$$
L(u)=\exp \left\{\int_{1}^{u} \frac{\varepsilon(t)}{t} d t\right\}, \quad \varepsilon(t) \rightarrow 0, \quad t \rightarrow \infty .
$$

Next, assume that $X_{i}=r_{i} \sin \alpha_{i}, 1-Y_{i}=r_{i} \cos \alpha_{i}$ and denote by $C_{n}$ the convex hull generated by random points $\left(X_{1}, Y_{1}\right),\left(X_{2}, Y_{2}\right), \ldots,\left(X_{n}, Y_{n}\right)$, and denote by $\nu_{n}, s_{n}$ and $l_{n}$ the number of vertices, the area and the perimeter of the $C_{n}$, respectively.

Denoting the largest root of the equation by $b_{n}$

$$
n x^{-\left(\beta+\frac{1}{2}\right)} L(x)=1 .
$$

In this case, in [2] obtained asymptotic expressions for the expectations of $E \nu_{n}, E s_{n}$ and $E l_{n}$. In the one-dimensional case, in [13] studied the role of the extreme summands in the sums, when the tail of the distribution of the initial random variable is (1) regularly varying. This paper is a continuation of $[9,10,13]$ in the multidimensional case. According to P. Groeneboom's remark, we consider $\nu_{n}$ for the case when $L(x)=1$. Then from (2) we get

$$
b_{n}=n^{\frac{2}{2 \beta+1}} .
$$

The basic theorem of the present paper is given.

Theorem 1. Let the conditions At $n \rightarrow \infty$ the following ratio is true

$$
\frac{\nu_{n}-a_{1}(\beta, n) b_{n}^{\frac{1}{2}}}{a_{2}(\beta, n) b_{n}^{\frac{1}{4}}} \stackrel{d}{\Rightarrow} \mathrm{N}(0,1) .
$$

Here $\stackrel{d}{\Rightarrow}$ means the weak convergence, $\mathrm{N}(0,1)$ denotes the standard normal distribution with parameters $(0,1), a_{1}(\beta, n), a_{2}(\beta, n)$ are positive constants determined from relations (12) and (13).

In particular, if $\beta=1$ Groeneboom's result [4] follows.

Corollary. If condition (3) is satisfied, then

$$
\frac{\nu_{n}-c_{1}(\beta) b_{n}^{\frac{1}{2}}}{c_{2}(\beta) b_{n}^{\frac{1}{4}}} \stackrel{d}{\Rightarrow} \mathrm{N}(0,1),
$$

where $c_{1}(\beta), c_{2}(\beta)$ have an explicit form, and $c_{1}(1), c_{2}(1)$ coincide with the corresponding constants in the [4]. 


\section{Preliminaries}

In this section we give a modification of the key approach by $[4,12]$ on the Poisson approximation of binomial point process (b.p.p.) $\mathrm{B}_{n}(\cdot)$ generated by $n$ random sample from the distribution (1) in the unit disk.

Assume that

$$
\begin{aligned}
& S_{\varepsilon}=\left\{(x, y): 1-\varepsilon \leqslant \sqrt{x^{2}+(1-y)^{2}} \leqslant 1\right\}, \\
& \Lambda_{\beta}(A)=P\left(\left(X_{1}, Y_{1}\right) \in A\right) .
\end{aligned}
$$

Consider a convex hull $C_{n}^{\prime}$ generated by a nonhomogeneous Poisson point process (n.h.p.p.p.) $\Pi_{n}\left(S_{\varepsilon}\right)$ with intensity of $n \Lambda_{\beta}(\cdot)$.

Lemma 1. Let $\mathrm{B}_{n}\left(S_{\varepsilon}\right)$ be the n.h.b.p.p. with parameter $\left(n, \Lambda_{\beta}(\cdot)\right)$. Then there is the n.h.p.p.p. $\Pi_{n}\left(S_{\varepsilon}\right)$ with intensityn $\Lambda_{\beta}(\cdot)$ such that

$$
P\left(\mathrm{~B}_{n}\left(S_{\varepsilon}\right) \neq \Pi_{n}\left(S_{\varepsilon}\right)\right) \leqslant 2 \Lambda_{\beta}\left(S_{\varepsilon}\right), \quad P\left(C_{n} \neq C_{n}^{\prime}\right) \rightarrow 0,
$$

at $n \rightarrow \infty, \varepsilon \rightarrow 0$.

To formulate Lemma 2, we need some notation.

Let $R(\delta)=\left\{(x, y): y<1,1-\frac{\delta^{2}}{2} \leqslant \sqrt{x^{2}+(1-y)^{2}} \leqslant 1,\left|\frac{x}{1-y}\right|<\operatorname{tg} \delta\right\}$,

$$
R^{*}(\delta)=\left\{(x, y):|x| \leqslant \delta, \frac{x^{2}}{2} \leqslant y \leqslant \frac{x^{2}}{2}+\frac{\delta^{2}}{2}\right\},
$$

where $\delta=\delta_{n}=c \sqrt{\log n / b_{n}}$.

For any set of forms

$$
A=\left\{(x, y): a \leqslant x \leqslant b, \frac{x^{2}}{2} \leqslant y_{1}(x) \leqslant y \leqslant y_{2}(x)\right\}
$$

introduce a measure

$$
\begin{aligned}
& \Lambda^{*}(A)=\frac{n \beta}{2 \pi} \int_{a}^{b} d x \int_{y_{1}(x)}^{y_{2}(x)}\left(y-\frac{x^{2}}{2}\right)^{\beta-1} d y, \\
& \Lambda^{*}(B)=0, \quad \text { if } B \subset\left\{(x, y): y<\frac{x^{2}}{2}\right\} .
\end{aligned}
$$

Then assume that

$$
\Lambda(B)=0, \text { at } B \subset\left\{(x, y): x^{2}+(1-y)^{2}>1\right\} .
$$

Lemma 2. There is $\Pi_{n}^{*}\left(S_{\varepsilon}\right)$ the n.h.p.p.p. with intensity $\Lambda_{n}^{*}(\cdot)$ such that for any $\varepsilon>0$

$$
P\left(\Pi_{n}^{*}\left(S_{\varepsilon}\right) \neq \Pi\left(S_{\varepsilon}\right)\right)=o\left(\frac{\log ^{\beta+2} n}{b_{n}^{1-\varepsilon}}\right) .
$$

We denote by $C_{n}^{*}$ the convex hull generated by the realization of n.h.p.p.p. $\Pi_{n}^{*}(D)$, where

$$
D=\left\{(x, y): \frac{x^{2}}{2} \leqslant y \leqslant 1\right\}, \quad S^{*}(\varepsilon)=\left\{(x, y): \frac{x^{2}}{2} \leqslant y \leqslant \frac{x^{2}}{2}+\varepsilon, y \leqslant 1\right\} .
$$


Then assume that

$$
R_{n}=\left\{(x, y): \frac{x^{2}}{2 b_{n}} \leqslant y\right\} .
$$

Introduce the following measure

$$
\Lambda_{n}^{0}(A)=\left\{\begin{array}{cc}
-\frac{1}{2 \pi \sqrt{b_{n}}} \iint_{A}\left(y-\frac{x^{2}}{2}\right)^{\beta-1} d x d y, A \subset R_{n}, \\
0, & \text { at } A \not \subset R_{n} .
\end{array}\right.
$$

Then denoting by $\Pi_{n}(\cdot)$ n.h.p.p.p. with intensity $\Lambda_{n}^{0}(\cdot)$ it is easy to see that

$$
\Pi_{n}(\cdot) \stackrel{d}{=} \Pi_{n}^{*}\left(b_{n}^{-1} \cdot\right) \text {. }
$$

Now the whole circle is divided by $m_{n}$ parts, where $m_{n}=\sqrt{b_{n}} / \log n$. Each section is $2 \pi \sqrt{b_{n}} \log n$ long, with central angle $2 \pi \log n / \sqrt{b_{n}}$. Disk section corresponding to the circumference section

$$
\left(\pi \sqrt{b_{n}}(\log n)(2 k-1), \pi \sqrt{b_{n}}(\log n)(2 k+1)\right)
$$

is denoted by $I_{k, n},\left(k=0,1, \ldots, m_{n}-1\right)$.

From Lemma 2, Poisson's processes $\Pi_{n}^{*}\left(b_{n}^{-1} \cdot\right)$ and $\Pi_{n}^{0}(\cdot)$ are almost similar. So consider Poisson's process $\Pi_{n}^{0}(\cdot)$ in $I_{k, n}$ only.

Following [4], consider the statement of Poisson point process in each sector $I_{k, n}$ separately.

The vertex process $W_{n}(a)=\left(X_{n}(a), Y_{n}(a)\right)$ for any $a \in\left(a_{-}, a_{+}\right)$is such a point $\left(X_{k}, Y_{k}\right)$ of n.h.p.p.p. realization $\Pi_{n}^{0}(\cdot)$, for which $Y_{k}-a X_{k}$ takes the minimum value, where $a_{-}=$ $=-\pi \log n / \sqrt{b_{n}}, a_{+}=\pi \log n / \sqrt{b_{n}}$.

It is easy to understand from the definition that, $W_{n}(a)$ is a non-stationary Markov jump process.

The following lemma gives the types of distributions $W_{n}(a)$ which correspond to various situations.

Lemma 3. Let $s=y-a x+a^{2} b_{n} / 2$.

Then

$$
\begin{aligned}
& \text { 1) } P\left(W_{n}(0) \in(d x, d y)\right)=\frac{\beta}{2 \pi \sqrt{b_{n}}} \exp \left\{-\frac{y^{\beta+\frac{1}{2}}}{\sqrt{2} \pi} B\left(\beta+1 ; \frac{1}{2}\right)\right\}\left(y-\frac{x^{2}}{2 b_{n}}\right)^{\beta-1} d x d y \text {; } \\
& \text { 2) } P\left(W_{n}(0) \in(d x, d y)\right)=\frac{\beta}{2 \pi \sqrt{b_{n}}} \exp \left\{-\frac{s^{\beta+\frac{1}{2}}}{\sqrt{2} \pi} B\left(\beta+1 ; \frac{1}{2}\right)\right\}\left(y-\frac{x^{2}}{2 b_{n}}\right)^{\beta-1} d x d y \text {; } \\
& \text { 3) } P\left(W_{n}(a)=W_{n}(0) / W_{n}(0)=(x, y)\right)= \\
& =\exp \left\{-\frac{1}{2 \pi \sqrt{b_{n}}} \int_{x-a b_{n}}^{\sqrt{2 b_{n} s}}\left(s-\frac{u^{2}}{2 b_{n}}\right)^{\beta} d u-\int_{x}^{\sqrt{2 b_{n} y}}\left(y-\frac{u^{2}}{2 b_{n}}\right)^{\beta} d y\right\} .
\end{aligned}
$$

Proof. Let $v=a(u-x)+y$ be a straight line passing through points $(x, y)$ with angular coefficient $a, A(a, x, y)$ is the set of points in the domain bounded by lines $v=a(u-x)+y$ and $v=u^{2} /\left(2 b_{n}\right)$.

It is easy to see that if $u_{1}$ and $u_{2}$ are the roots of equation

$$
u^{2} /\left(2 b_{n}\right)=a(u-x)+y,
$$

then $u_{1,2}=a b_{n}+\sqrt{2 b_{n} s}$. 
Calculate $\Lambda_{n}^{0}(A(a, x, y))$ (see $\left.(5)\right)$. Considering

$$
y+a(u-x)-\frac{u^{2}}{2 b_{n}}=y-a x+\frac{a^{2} b_{n}}{2}-\frac{\left(u-a b_{n}\right)^{2}}{2 b_{n}}=s-\frac{\left(u-a b_{n}\right)^{2}}{2 b_{n}}
$$

we get

$$
\begin{aligned}
\Lambda_{n}^{0}(A(a, x, y)) & =\frac{1}{2 \pi \sqrt{b_{n}}} \int_{u_{1}}^{u_{2}}\left[y+a(u-x)-\frac{u^{2}}{2 b_{n}}\right]^{\beta} d u=\frac{1}{2 \pi \sqrt{b_{n}}} \int_{-\sqrt{2 b_{n} s}}^{\sqrt{2 b_{n} s}}\left(s-\frac{u^{2}}{2 b_{n}}\right)^{\beta} d u= \\
& =\frac{\sqrt{2} s^{\beta+\frac{1}{2}}}{\pi} \int_{0}^{1}\left(1-u^{2}\right)^{\beta} d u=\frac{s^{\beta+\frac{1}{2}}}{\pi \sqrt{2}} B\left(\beta+1 ; \frac{1}{2}\right) .
\end{aligned}
$$

Next, let $d=\sqrt{(\Delta x)^{2}+(\Delta y)^{2}}, \quad v=a u+c_{-}, \quad v=a u+c_{+}$be two straight lines parallel to $v=a(u-x)+y$ and passing at distanced from below and above, respectively.

By $A_{d}^{-}(a, x, y)$ and $A_{d}^{+}(a, x, y)$ denote the sets bounded by lines $v=a u+c_{-}, v=u^{2} /\left(2 b_{n}\right)$ and $v=a u+c_{+}, v=u^{2} /\left(2 b_{n}\right)$ respectively. Assume that $\Delta_{x, y}=[x, x+\Delta x] \times[y, y+\Delta y]$.

It follows from the definition $W_{n}(a)$ that if $\bar{\pi}(A)$ is the number of points in $A$ realization of n.h.p.p.p. $\Pi_{n}(\cdot)$, then

$$
P\left(W_{n}(a) \in \Delta_{x, y}\right) \leqslant P\left(\bar{\pi}\left(\Delta_{x, y}\right) \geqslant 1, \bar{\pi}\left(A_{d}^{-}(a, x, y)\right)=0\right) .
$$

On the other hand, it is easy to see that

$$
P\left(W_{n}(a) \in \Delta_{x, y}\right) \geqslant P\left(\bar{\pi}\left(\Delta_{x, y}\right)=1, \bar{\pi}\left(A_{d}^{+}(a, x, y)-\Delta_{x, y}\right)=0\right) .
$$

Considering the property of the Poisson process (the independence of increments) and from inequalities (7) and (8), using (5) at $d \rightarrow 0$ we obtain the first relation of the lemma.

Similarly, the other relations of the lemma are obtained.

Assume that

$$
R_{n}(a)=X_{n}(a)-a b_{n}, \quad S_{n}(a)=Y_{n}(a)-\frac{X_{n}^{2}(a)}{2 b_{n}}+\frac{R_{n}^{2}(a)}{2 b_{n}}, \quad T_{n}(a)=\left(R_{n}(a), S_{n}(a)\right) .
$$

Obviously that $T_{n}(0)=W_{n}(0)$ a.s. and therefore

$$
P\left(T_{n}(0) \in(d r, d s)\right)=P\left(W_{n}(0) \in(d r, d s)\right) .
$$

Lemma 3 leads to the following lemma.

Lemma 4. $T_{n}(a)$ is a stationary Markov jump process and

1) $P\left(T_{n}(0) \in(d r, d s)\right)=\frac{\beta}{2 \pi \sqrt{b_{n}}} \exp \left\{-\frac{s^{\beta+\frac{1}{2}}}{\sqrt{2} \pi} B\left(\beta+1 ; \frac{1}{2}\right)\right\}\left(s-\frac{r^{2}}{2 b_{n}}\right)^{\beta-1} d r d s$;

2) $P\left(T_{n}(a)=\left(r_{1}, s_{1}\right) / T_{n}(0)=\left(r_{0}, s_{0}\right)\right)=$

$$
=\exp \left\{-\frac{1}{\sqrt{2} \pi L\left(b_{n}\right)}\left[s_{1}^{\beta+\frac{1}{2}} \int_{\frac{r_{1}}{\sqrt{2 b_{n} s_{1}}}}^{1}\left(1-t^{2}\right)^{\beta} d t-s_{0}^{\beta+\frac{1}{2}} \int_{\frac{r_{0}}{\sqrt{2 b_{n} s_{0}}}}^{1}\left(1-t^{2}\right)^{\beta} d t\right]\right\},
$$

where $r_{1}=r_{0}-a b_{n}, s_{1}=s_{0}-a r_{0}+a^{2} b_{n} / 2$;

3) $P\left(T_{n}(a) \in\left(d r_{1}, d s_{1}\right) / T_{n}(0)=\left(r_{0}, s_{0}\right)\right)=P\left(T_{n}(a) \in\left(d r_{1}, d s_{1}\right)\right)$, 
if $a b_{n}-\sqrt{2 b_{n} s_{1}}>\sqrt{2 b_{n} s_{0}}$;

4) $P\left(T_{n}(a) \in\left(d r_{2}, d s_{2}\right) / T_{n}(0)=\left(r_{1}, s_{1}\right)\right)=$

$$
\begin{aligned}
& =\frac{1}{2 \pi \sqrt{b_{n}}} \exp \left\{-\frac{1}{\sqrt{2} \pi}\left[s_{2}^{\beta+\frac{1}{2}} \int_{\frac{s_{1}-s_{2}}{a \sqrt{2 b_{n} s_{2}}}+\frac{a b_{n}}{\sqrt{2 b_{n} s_{2}}}}^{1}\left(1-t^{2}\right)^{\beta} d t-\right.\right. \\
& \left.\left.-s_{1}^{\beta+\frac{1}{2}} \int_{\frac{s_{1}-s_{2}}{a \sqrt{2 b_{n} s_{1}}}+\frac{a b_{n}}{\sqrt{2 b_{n} s_{1}}}}^{1}\left(1-t^{2}\right)^{\beta} d t\right]\right\}\left(s_{2}-\frac{r_{2}^{2}}{2 b_{n}}\right)^{\beta-1} d r_{2} d s_{2} .
\end{aligned}
$$

Here assume that

$$
\begin{gathered}
\left(r_{i}, s_{i}\right) \in D=\left\{(r, s): s \geqslant\left(r^{2}\right) /\left(2 b_{n}\right)\right\}, \quad a b_{n}-\sqrt{2 b_{n} s_{2}} \leqslant \sqrt{2 b_{n} s_{1}}, \\
s_{2}+\left(a^{2} b_{n}\right) / 2+a r_{2} \geqslant s_{1} \geqslant s_{2}-\left(a^{2} b_{n}\right) / 2+a r_{1} .
\end{gathered}
$$

Consider the following $\sigma$-algebras generated by process $T_{n}(a)$ :

$$
\Im_{n}^{0}=\sigma\left\{T_{n}(c): c \leqslant 0\right\}, \quad \Im_{n}^{a+}=\sigma\left\{T_{n}(c): c \geqslant a\right\} .
$$

From Lemmas 3 and 4 it is easy to prove the properties of strong mixing of the process $T_{n}(a)$

Lemma 5. For any $A \in \Im_{n}^{0}$ and $B \in \Im_{n}^{a+}|P(A \bigcap B)-P(A) P(B)| \leqslant \tau_{n}(a)$, where

$$
\tau_{n}(a) \leqslant 4 \exp \left\{-\frac{1}{\sqrt{2} \pi}\left(\frac{a^{2} b_{n}}{8}\right)^{\beta+\frac{1}{2}} B\left(\beta+1 ; \frac{1}{2}\right)\right\} \text {. }
$$

Lemma 6. If $a>\left(a_{n} \varepsilon_{n}^{*}\right) / b_{n}$, then under the conditions of Lemma 5 we have $\sum_{n=1}^{\infty}\left(\tau_{n}(a)\right)^{\tau}<\infty$ for any $\tau>0$, where $a_{n}=\sqrt{2 b_{n} \log n}, \varepsilon_{n}^{*}=(\log n)^{-\frac{2 \beta-\delta-1}{2(2 \beta+1)}}, 0<\delta<1$.

Proof. If $a>\left(a_{n} \varepsilon_{n}^{*}\right) / b_{n}$, then from Lemma 5 we get

$$
\tau_{n}(a) \leqslant 4 \exp \left\{-c(\log n)^{1+\frac{\delta}{3}}\right\} .
$$

This immediately implies the statement of Lemma 6.

Now introduce notations

$$
\begin{aligned}
M^{(k)}\left(t ; R^{2}\right) & =\frac{\beta}{2 \pi \sqrt{b_{n}}} \int_{r}^{\sqrt{2 b_{n} s}}(u-r)^{k}\left(s-\frac{u^{2}}{2 b_{n}}\right)^{\beta-1} d u= \\
& =\frac{\beta}{2 \pi \sqrt{b_{n}}} \int_{0}^{\sqrt{2 b_{n} s}-r} u^{k}\left(s-\frac{(u+r)^{2}}{2 b_{n}}\right)^{\beta-1} d u
\end{aligned}
$$

where $t=(r, s)$.

Lemma 7. Processes

$$
N(a)-\int_{0}^{a} M^{(1)}\left(T(b) ; R^{2}\right) d b
$$

and

$$
N^{2}(a)-\int_{0}^{a}[2 N(b)+1] M^{(1)}\left(T(b) ; R^{2}\right) d b
$$

are martingales with respect to $\sigma$-algebra $\Im_{[0, a]}=\sigma\{T(c): 0 \leqslant c \leqslant a\}$. 
Proof. We have

$$
E\left\{N(a+h)-N(a) / \Im_{[0, a]}\right\}=E\{N(a+h)-N(a) / T(a)\}
$$

Hence, due to stationary nature of the process $T(a)$

$$
\begin{gathered}
E\{N(a+h)-N(a) / T(a)=(r, s)\}=E\{N(h)-N(0) / T(0)=(r, s)\} \sim \\
\sim E \bar{\pi}\left(A^{*}(h ; r, s)\right) \sim \Lambda^{0}\left(A^{*}(h ; r, s)\right),
\end{gathered}
$$

where $\bar{\pi}\left(A^{*}(h ; r, s)\right)$ is defined in the previous paragraph and

$$
A^{*}(h ; r, s)=A^{0}(h ; r, s) \bigcup A^{1}(h ; r, s) .
$$

Further, by the definition $\Lambda^{0}(\cdot)$ of a measure (see $\left.(5)\right)$, it is easy to show that at small $h$ $\Lambda_{n}^{0}\left(A^{0}(h ; r, s)\right)=o(h)$ and

$$
\Lambda_{n}^{0}\left(A^{(1)}\left(a, b, x_{0}, y_{0}\right)\right) \leqslant C_{2} h^{\beta-\varepsilon} \int_{a b_{n}+\sqrt{2 b_{n} s_{0}}}^{b b_{n}+\sqrt{2 b_{n} s_{1}}} d u=O\left(h^{\beta+1-\varepsilon}\right)
$$

From the latter, again using definition (5) at small $h$, we have

$$
\Lambda_{n}^{0}\left(A^{*}(h ; r, s)\right)=\Lambda_{n}^{0}\left(A^{0}(h ; r, s)\right)+o(h)=h M^{(1)}\left(t ; R^{2}\right)+o(h) .
$$

By virtue of (9) and (10), we obtain the proof of the first statement of the lemma. Proceed to the proof of the second statement of Lemma 6.

We have

$$
\begin{gathered}
E\left\{N^{2}(a+h)-N^{2}(a) / T(a)=(r, s)\right\}= \\
=E\{(N(a+h)-N(a))(N(a+h)-N(a)+2 N(a)) / T(a)=(r, s)\}= \\
=E\left\{(N(a+h)-N(a))^{2} / T(a)=(r, s)\right\}+2 N(a) E\{N(a+h)-N(a) /-T(a)=(r, s)\}= \\
=E\{N(a+h)-N(a) / T(a)=(r, s)\}+o(h)+2 N(a) E\{N(a+h)-N(a) / T(a)=(r, s)\}= \\
=(2 N(a)+1) E\{N(a+h)-N(a) / T(a)=(r, s)\}+o(h)= \\
=(2 N(a)+1) h M^{(1)}\left(t, R^{2}\right)+o(h) .
\end{gathered}
$$

So, Lemma 7 is completely proved.

Using these Lemmas, calculate the asymptotic behavior of the moments $N(a)$ and $N^{2}(a)$ at fixed $a$ and at $n \rightarrow \infty$.

Let

$$
c_{0}=\left(B\left(2 \beta+1, \frac{1}{2}\right) / \sqrt{2}\right)\left(\frac{\sqrt{2} \pi}{B\left(2 \beta+1, \frac{1}{2}\right)}\right)^{2-\frac{1}{2 \beta+1}} \Gamma\left(3-\frac{1}{2 \beta+1}\right),
$$

where $B(\cdot, \cdot)$ and $\Gamma(\cdot)$ are the known beta and gamma functions, respectively.

Lemma 8. We have

$$
E N(a)=a \lambda_{n}^{(1)} \sqrt{b_{n}}, \quad D N(a)=a \lambda_{n}^{(2)} \sqrt{b_{n}}, \text { as } n \rightarrow \infty,
$$

where $\lambda_{n}^{(1)}=\frac{c_{0}}{\sqrt{2 \pi}}+o(1), \lambda_{n}^{(2)}=c_{0}+o(1)$. 
Proof. We use Lemma 7. Since the process $T(\cdot)$ is stationary, we have

$$
E N(a)=E \int_{0}^{a} M^{(1)}\left(T(b) ; R^{2}\right) d b=a E M^{(1)}\left(T(0) ; R^{2}\right) .
$$

By definition of $M^{(1)}\left(t ; R^{2}\right)$, after some identical transformations we have

$$
\begin{aligned}
E M^{(1)}\left(T(0) ; R^{2}\right)= & \frac{\beta}{2 \pi \sqrt{b_{n}}} E \int_{0}^{\sqrt{2 b_{n}}-r} u\left(s-\frac{(u+r)^{2}}{2 b_{n}}\right)^{\beta-1} d u= \\
= & \frac{\beta}{2 \pi \sqrt{b_{n}}} E\left\{\int_{0}^{\sqrt{2 b_{n} s}-r} u\left(s-\frac{(u+r)^{2}}{2 b_{n}}\right)^{\beta-1} d u\right\}= \\
= & \frac{\sqrt{2 b_{n}} \beta^{2}}{2 \pi^{2}}\left\{\int_{0}^{\infty} \exp \left[-\frac{B\left(\beta+1, \frac{1}{2}\right)}{\sqrt{2} \pi} s^{\beta+\frac{1}{2}}\right] s^{2 \beta-\frac{1}{2}} d s\right\} \times \\
& \times \int_{-1}^{1}\left\{\left(1-r^{2}\right)^{\beta-1} \int_{0}^{1-r} u\left(1-(u+r)^{2}\right)^{\beta-1} d u\right\} d r= \\
= & \frac{\sqrt{b_{n}} \beta^{2}}{2 \pi^{2}(4 \beta+1)}\left(\frac{\sqrt{2} \pi}{B\left(\beta+1 ; \frac{1}{2}\right)}\right)^{\frac{4 \beta+1}{2 \beta+1}} \cdot \Gamma\left(2-\frac{1}{2 \beta+1}\right) \times \\
& \times \int_{-1}^{1}\left(1-r^{2}\right)^{\beta-1} d r \int_{0}^{1-r} u\left(1-(u+r)^{2}\right)^{\beta-1} d u .
\end{aligned}
$$

It is easy to calculate that

$$
\int_{-1}^{1}\left(1-r^{2}\right)^{\beta-1} d r \int_{0}^{1-r} u\left(1-(u+r)^{2}\right)^{\beta-1} d u=\frac{1}{\beta} \int_{0}^{1} \frac{t^{2 \beta+1} d t}{\sqrt{1-t}}=\frac{B\left(2 \beta, \frac{1}{2}\right)}{\beta} .
$$

From relations (11)-(13), the proof of the statement of the first part of Lemma 8 follows.

The second part of Lemma 8 is easy to prove, using Lemma 7 and the first part of Lemma 8 , and Lemma 2.6 considering in [4].

\section{Proof of the theorem}

Assume that $N_{k, m_{n}}^{*}$ is the number of vertices of the convex hull in $I_{k, m_{n}}^{*}$ - "big block" and $N_{k, m_{n}}^{* *}$ is the number of vertices of the convex hull in $I_{k, m_{n}}^{* *}-$ "small block", where sectors $I_{k, m_{n}}^{*}$ and $I_{k, m_{n}}^{* *}$ correspond to

$$
\left(\pi \sqrt{b_{n}}(\log n)(2 k-1)+\xi_{n}, \pi \sqrt{b_{n}}(\log n)(2 k+1)-\xi_{n}\right)
$$

and

$$
\left(\pi \sqrt{b_{n}}(\log n)(2 k+1)-\xi_{n}, \pi \sqrt{b_{n}}(\log n)(2 k+1)+\xi_{n}\right)
$$

of the part of disk, respectively and

$$
\xi_{n}=\pi \sqrt{b_{n}}(\log n)^{1-\delta}, \quad 0<\delta<1 /[2(2 \beta+1)] .
$$

Hence

$$
\nu_{n}=\sum_{k=0}^{m_{n}-1} N_{k, m_{n}}^{*}+\sum_{k=0}^{m_{n}-1} N_{k, m_{n}}^{* *} .
$$


By the principle of construction of sectors $I_{k, m_{n}}^{*}$ and $I_{k, m_{n}}^{* *}, N_{k, m_{n}}^{* *}$ is insignificant relative to $N_{k, m_{n}}^{*}$. Further, from Lemmas 5 and Theorem 17.2.2 in [8], we can apply the classical central limit theorem for the summs of random variables $N_{0, m_{n}}^{*}+N_{1, m_{n}}^{*}+\cdots+N_{m_{n}-1, m_{n}}^{*}$ and $N_{0, m_{n}}^{* *}+$ $+N_{1, m_{n}}^{* *}+\cdots+N_{m_{n}-1, m_{n}}^{* *}$. Therefore, we get that

$$
\begin{gathered}
\frac{\nu_{n}-m_{n}\left(E N_{k, m_{n}}^{*}+E N_{k, m_{n}}^{* *}\right)}{\sqrt{m_{n} D N_{k, m_{n}}^{*}}}=\frac{1}{\sqrt{m_{n}}} \sum_{k=0}^{m_{n}-1} \frac{N_{k, m_{n}}^{*}-E N_{k, m_{n}}^{*}}{\sqrt{D N_{k, m_{n}}^{*}}}+ \\
+\frac{1}{\sqrt{m_{n}}} \sum_{k=0}^{m_{n}-1} \frac{N_{k, m_{n}}^{* *}-E N_{k, m_{n}}^{* *}}{\sqrt{D N_{k, m_{n}}^{* *}}} \frac{\sqrt{D N_{k, m_{n}}^{* *}}}{\sqrt{D N_{k, m_{n}}^{*}}}+o(1)= \\
=\frac{1}{\sqrt{m_{n}}} \sum_{k=0}^{m_{n}-1} \frac{N_{k, m_{n}}^{*}-E N_{k, m_{n}}^{*}}{\sqrt{D N_{k, m_{n}}^{*}}}+\frac{1}{\sqrt{m_{n}}} \sum_{k=0}^{m_{n}-1} \frac{N_{k, m_{n}}^{* *}-E N_{k, m_{n}}^{* *}}{\sqrt{D N_{k, m_{n}}^{* *}}} \cdot o(1)+o(1) \stackrel{d}{\Rightarrow} \mathrm{N}(0,1) .
\end{gathered}
$$

The theorem is proved.

\section{References}

[1] A.J.Cabo, P.Groeneboom, Probab. Theory Relat. Fields, 100(1994), 31-55. DOI: $10.1007 / \mathrm{BF} 01204952$

[2] H.Carnal, Z. Wahrscheinlichkeits theorie verw. Geb., 15(1970), 168-176. DOI: $10.1007 / \mathrm{BF} 00531885$

[3] B.Efron, Biometrika, 52(1965), 331-343. DOI: 10.1093/biomet/52.3-4.331

[4] P.Groeneboom, Probab. Theory Related Fields, 79(1988), 327-368. DOI: $10.1007 / \mathrm{BF} 00342231$

[5] P.Groeneboom, Convex hulls of uniform samples from a convex polygon. Adv. Appl. Prob. (SGSA), 44(2012), 330-342. DOI: 10.1239/aap/1339878714

[6] ] T.Hsing, On the asymptotic distribution of the area outside a random convex hull in a disk, The Annals of Applied Probability, 4(1994), no. 2, 478-493.

[7] I.Hueter, The convex hull of a normal sample, Adv. Appl. Prob., 26(1994), 855-875.

[8] I.A.Ibragimov, Yu.U.Linnik, Independent and stationary sequences of random variables, Izdatelstvo Nauka, Moscow, 1965 (in Russian).

[9] I.M.Khamdamov, T.Kh.Adirov, Martingale properties of vertex functionals generated by Poisson point processes, Reports of the Academy of Sciences of Uzbekistan, (2015), no. 1, 9-11 (in Russian).

[10] I.M.Khamdamov, T.Kh.Adirov, One of the properties of the convex hull generated by a Poisson point process, Uzbek Mathematical Journal, (2019), no. 3, 60-63.

[11] A.V.Nagaev, Some properties of convex hulls generated by homogeneous Poisson point processes in an unbounded convex domain, Ann. Inst. Statist. Math., 47(1995), no. 1, 21-29. 
[12] A.V.Nagaev, I.M.Khamdamov, Limit theorems for functionals of random convex hulls, Preprint of Institute of Mathematics, Academy of Sciences of Uzbekistan, Tashkent, 1991 (in Russian).

[13] A.V.Nagaev, I.M. Khamdamov, On the Role of Extreme Summands in Sums of Independent Random Variables, Theory of Probability and Its Applications, 47(2003), no. 3, 533-541.

[14] H.Raynaud, Sur l'enveloppe convexe des nuages de points aleatoires dans, J. Appl. Prob., 7(1970), 35-48.

[15] A.Reny, R.Sulanke, Uber diekovexe Hulle von zufalling gewahlten Punkten, Z. Wahrscheinlichkeits theorie verw. Geb., 2(1963), 75-84.

\title{
О предельной теореме для числа вершин выпуклых оболочек в единичном круге
}

Исакжан М. Хамдамов

Ташкентский университет информационных технологий

\begin{abstract}
Аннотация. Данная статья посвящена дальнейшему исследованию свойства ряда вершин выпуклых оболочек, порожденных независимыми наблюдениями двумерного случайного вектора с регулярными распределениями вблизи границы носителя, когда он является единичным диском. Следуя П. Гренебуму [4], биномиальный точечный процесс аппроксимируем пуассоновским точечным процессом вблизи границы опоры и строим вершинные процессы выпуклых оболочек. Исследованы свойства сильного перемешивания и мартингальности вершинных процессов. Используя эти свойства, получаем асимптотические выражения для ожиданий и дисперсии вершинных процессов, которые соответствуют результатам, ранее полученным Н. Карнала [2]. Далее, используя свойства сильного перемешивания вершинных процессов, доказываем центральную предельную теорему для ряда вершин выпуклой оболочки.
\end{abstract}

Ключевые слова: выпуклая оболочка, пуассоновский точечный процесс, скачкообразный марковский процесс, мартингальность, центральная предельная теорема. 